\title{
ZONIFICACIÓN POR RIESGO DE EXPLOSIONES DE GAS METANO EN LA MINERÍA DEL CARBÓN EN BOYACÁ, COLOMBIA
}

\section{Zoning for risk of methane gas explosions in coal mining in Boyacá, Colombia}

\author{
Juan Sebastián Arteaga Hernández \\ Escuela de Ingeniería Geológica, Universidad Pedagógica y Tecnológica de Colombia, Sede Sogamoso \\ Email: juan.arteaga@uptc.edu.co
}

(Recibido el 06 de febrero de 2021 - Aceptado el 09 de junio de 2021)

\section{Resumen}

En Boyacá el número de muertes por explosiones de metano en minas de carbón constituye el mayor riesgo minero, y las víctimas pueden estar alrededor de 10 muertos por año. Por lo tanto, se hizo zonificación del departamento para determinar las zonas mineras con mayor riesgo a partir de las mediciones de las concentraciones de metano en los frentes mineros. De la base de datos de la Agencia Nacional de Minería, se revisaron los contenidos de gas metano reportados en porcentaje durante las visitas de fiscalización. Dicha información se organizó y tabulo en una plataforma SIG, luego se filtró para utilizar los datos de mayor utilidad. Este rastreo arrojó que la zona de mayor riesgo está entre Tasco y Socotá, donde también se han reportado los mayores contenidos de gas en los ensayos de desorción; por lo tanto, este sector requiere mayor atención y cuidado durante las visitas de seguimiento a fin de evitar accidentes.

Palabras claves: Colombia, carbón, minería, metano, explosiones, SIG.

\begin{abstract}
In Boyacá, the number of deaths from methane explosions in coalmines constitutes the greatest mining risk, and the victims can be around 10 deaths per year. Therefore, the province was zoning to determine the mining areas with the highest risk from the measurements of methane concentrations on the mining fronts. From the database of the National Mining Office, the methane gas contents reported in percentage during the inspection visits were reviewed. This information was organized and tabulated in a GIS platform, then filtered to use the most useful data. This review shows that the area of greatest risk is between Tasco and Socotá, where the highest gas contents have also been reported in desorption tests; based on that, this area requires more attention and care during follow-up visits in order to avoid accidents.
\end{abstract}

Keywords: Colombia, coal, mining, methane, explosions, GIS.

\section{INTRODUCCIÓN}

En el departamento de Boyacá, el número de muertes por explosiones de metano en minas de carbón constituye el mayor riesgo minero, y las víctimas pueden estar alrededor de 10 muertos por año. Este reporte es alarmante ya que las cifras no disminuyen con el tiempo, a pesar de que el reglamento de seguridad en labores subterráneas establece que los porcentajes máximos permisibles de metano en los frentes de explotación de carbón son de 1 \% (Decreto 1886 de 1915 del Ministerio de Minas y Energía) [1]. Además de la presencia del metano en 
porcentajes mayor al $1 \%$, también se requiere un agente de ignición; porque las explosiones pueden iniciarse por distintas fuentes como lo son: las llamas abiertas, cortos circuitos, circuitos eléctricos no sellados intrínsecamente, descargas eléctricas y electricidad estática.

Algunos investigadores han reconocido el problema y han investigado al respecto. Mariño et al. [2] encontraron que, aunque las compañías mineras hacen un seguimiento continuo de los contenidos de gases con ayuda de multidetectores, desconocen los contenidos reales del gas en el carbón in situ; por lo tanto, el planeamiento minero y demás medidas de prevención se estarían haciendo sobre valores relativos de contenido de gas.

La UPME [3] indica que las cifras por accidentes son altas y que el drenaje o utilización previa del gas metano en las minas de carbón ayudaría a disminuir los accidentes. El grupo de investigación en minería de la Universidad Nacional - Sede Medellín realizó mediciones de concentraciones de metano durante el arranque en minas a lo largo de la cuenca del Sinifaná, a partir de las cuales se elaboraron perfiles y correlaciones para determinar los factores más influyentes para las acumulaciones de gas y por ende explosiones [4]. Finalmente, Mariño y Bedoya determinaron los contenidos de gas en las minas de carbón a diferentes profundidades a partir de los ensayos de desorción cánisters de muestras obtenidas en perforaciones [5].

Aunque se tiene una idea de los contenidos de gas a partir de perforaciones utilizando ensayos de desorción, y se conocen algunas generalidades sobre los riesgos de explosión en la minería del carbón, no se tiene un análisis sistemático de las mediciones con metanómetros en la atmósfera de los frentes de las minas que establezca cuáles son las zonas con más riesgo; por lo tanto, el propósito de esta investigación es zonificar el departamento de Boyacá, mediante un polígono, donde se presenten las áreas y zonas mineras con mayor riesgo a partir de las concentraciones de metano en los frentes, y así generar un planteamiento nuevo para el análisis del problema, de tal manera que, se enfoque y mejore la efectividad de las visitas de fiscalización (Figura 1).

Los objetivos que enmarcan esta ruta investigativa son: organizar y tabular los datos que se tienen para el análisis, filtrar los datos más representativos, procesar lo datos y, mediante interpolación, presentar las zonas más representativas en cuanto a riesgo por explosión. Finalmente, delimitar las zonas vulnerables por explosión de gas metano.

Las limitaciones de este estudio están dadas por la información disponible, pues esta es limitada, de acuerdo al contenido de metano en los frentes de las minas; en contraste, a la reportada durante las visitas de fiscalización.

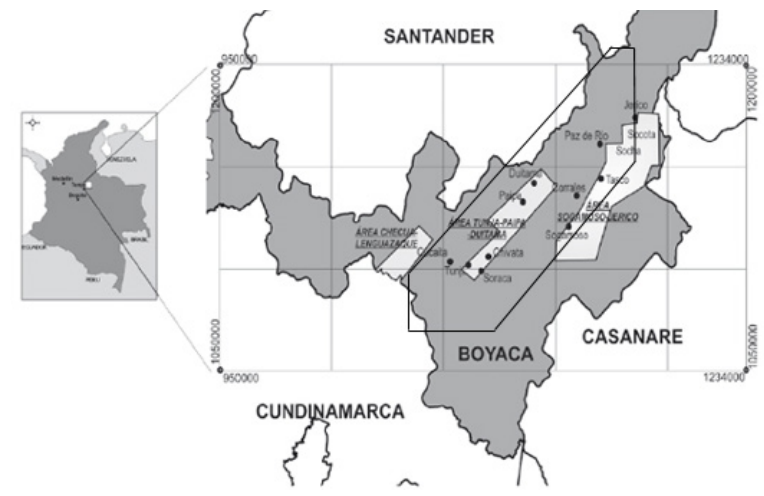

Figura 1. Ubicación de dos áreas carboníferas estudiadas en Boyacá, y del polígono de la zona de estudio.

\section{MARCO TEÓRICO SOBRE EL GAS EN EL CARBÓN}

El gas asociado al carbón (CBM) está compuesto aproximadamente en un 80 \% o más de metano; el resto, por lo general, suele ser dióxido de carbono $\left(\mathrm{CO}_{2}\right)$, nitrógeno $\left(\mathrm{N}_{2}\right)$ y etano. El gas asociado al carbón también conocido como gas de los mantos de carbón, CBM o CBG por sus siglas en inglés (GMAC, CBG o GAC en español), es una fuente de gas no convencional, que se obtiene a partir de la extracción del metano contenido en las capas de carbón. El CBM se genera en el carbón durante el proceso de carbonización de la materia orgánica por incrementos de presión y temperatura producto de los procesos geológicos. También incluye el gas que se 
origina en el carbón por procesos bioquímicos, debido a la intervención de bacterias [2].

Las altas presiones debajo de la superficie conservan el gas metano en la matriz del carbón en un estado de adsorción y absorción. Estas altas presiones son creadas tanto por la profundidad, como por el agua que está contenida en la matriz del carbón. Para liberar el metano, los pozos productores de los mantos de carbón o los túneles mineros permiten que el agua sea bombeada para bajar la presión, lo que ayuda a desorber el gas del carbón. El metano migra dentro y fuera de los mantos de carbón hasta encontrar una barrera que lo atrape; de lo contrario, continúa migrando hasta alcanzar la superficie terrestre $y$, finalmente, se incorpora a la atmósfera como gas de efecto invernadero (GEI).

Los mantos de carbón más profundos son extraídos a través de túneles y labores subterráneas, mejor conocidas como "minería subterránea" como la que se trabaja en el Altiplano Cundiboyacense, Norte de Santander y Antioquia. Por la profundidad a la que se realizan las actividades mineras subterráneas, estas tienden a emitir más metano que las minas a cielo abierto. Además de la profundidad, el contenido de gas en el carbón se determina por 4 variables adicionales: 1) rango del carbón (grado de maduración termal), 2) tipo de carbón (composición maceral), 3) Contenido de materia mineral, y 4) espesor de la cobertera e historia del enterramiento y subsidencia.

El contenido del gas en el carbón se puede determinar a partir de métodos indirectos y directos. Los métodos indirectos no miden el gas en la capa de carbón, sino que se infiere a partir de alguna variable como la calidad del carbón, la profundidad, la reflectancia de la vitrinita o las isotermas de adsorción. Dichos métodos suponen la presencia del gas, pero no lo demuestran directamente. Los métodos directos se dividen en dos grandes grupos:

1) la medición relativa del contenido en la atmósfera de la mina utilizando metanómetros que miden el porcentaje de metano en la ventilación de la mina, tal como se midieron los resultados de la presente investigación, y 2) medición absoluta del contenido de metano en los mantos de carbón sobre corazones de perforación que se introducen en contenedores herméticos llamados cánister, el contenido del gas se mide por desplazamiento de una columna de agua y se conocen como ensayos de desorción. En este caso los contenidos se reportan en $\mathrm{m}^{3}$ / ton o pies³ de gas por tonelada de carbón [2].

Como el gas metano es explosivo, se deben medir y conocer muy bien los contenidos de metano en cada macizo rocoso a fin de diseñar el planeamiento minero sobre esos valores y evitar accidentes; por ejemplo, en Boyacá, a partir de ensayos de desorción se determinó que entre 0 y $200 \mathrm{~m}$ de profundidad el contenido de gas estaría entre 10 y $50 \mathrm{pies}^{3} /$ ton, y que entre 200 y $400 \mathrm{~m}$ de profundidad el contenido de gas estaría entre 50 y 130 $\mathrm{pies}^{3} /$ ton que equivalen a 1,41 y $3,68 \mathrm{~m}^{3} /$ ton [5].

La búsqueda de la información y el análisis se hizo en 2 de las 10 áreas carboníferas existentes en Boyacá: área carbonífera Tunja-Paipa-Duitama (802), y área carbonífera Sogamoso- Jericó (803) [6]. A continuación, se presentan los aspectos más relevantes por área carbonífera.

\section{Área carbonifera Tunja-Paipa-Duitama (802)}

La estructura principal del área es el Sinclinal Tunja-Paipa, con dirección suroeste-noreste. En donde, asociados a esta estructura, se desarrollan pliegues anticlinales y sinclinales con dirección similar. En el área 802 la formación Guaduas se ha dividido en tres miembros que de base a techo se conocen como miembros Inferior (110 m), Medio (210 m) y Superior (210 m). El miembro medio contiene los carbones y está comprendido desde el manto pequeño hasta un banco de arenisca de grano medio, con un espesor de $30 \mathrm{~m}$, que aflora en la mayor parte del área y que sirve como nivel guía. Esta unidad está compuesta por arcillolitas grises, intercaladas con areniscas de grano fino y hasta nueve mantos de carbón [7] [8].

Los contenidos de gas encontrados en las perforaciones adelantadas en Chivatá, Tunja, Cucaita, Soracá y Paipa son muy bajos y fluctúan entre 0 y $65 \mathrm{pie}^{3} /$ ton a profundidades 
entre 35 y 350 m; los pozos con mayores contenidos se encontraron en Chivatá y Cucaita con 33 y 65 pie $^{3} /$ ton, respectivamente, en las mejores muestras [9]. Los bajos contenidos de gas se pueden explicar a partir de la cercanía de los carbones a la superficie y al intenso fracturamiento en la zona, producto de la prolongación de la Falla de Boyacá, que ha impedido el desarrollo de una industria extractiva de carbón en las cercanías a Tunja [10] [8].

Área carbonífera Sogamoso - Jericó (803)

El área se extiende sobre la parte centro - nororiental del departamento de Boyacá, entre Cuítiva en el sur y Jericó en el norte (Figura 1). El espesor de la Formación Guaduas disminuye de 480 m en Sogamoso a 350 m en Socotá. En el área la formación ha sido dividida en dos conjuntos: Superior, constituido por una serie continua de intercalaciones de limonitas, lutitas grises, arenitas de cuarzo y, entre seis y ocho mantos de carbón explotables, con un espesor de 300 m; en el Inferior, no se encuentran mantos de carbón de suficiente espesor para desarrollar minería y consta de lodolitas y arcillolitas grises oscuras a negras prevalecientes sobre intercalaciones de arenitas [8].

Los valores encontrados en los frentes de minas de carbón del municipio de Sogamoso son bajos (0-2 $\mathrm{pie}^{3} /$ ton) debido al muestreó en frentes de minas relativamente superficiales. Los mayores contenidos de gas en Sogamoso se encontraron en los Bloques Chiguatá y Morcá con valores que están entre 1,9 y 1,6 pie $^{3}$ /ton y esto se explica porque estos bloques hacen parte de un sinclinal y están menos fallados [11] [12] [8].

En una perforación hacia el sur de Sogamoso, que alcanzó los $150 \mathrm{~m}$, se encontraron valores entre 1 y $6 \mathrm{pie}^{3} /$ ton, lo que se considera bajo para dichas profundidades, como consecuencia del intenso fallamiento, ya que se encontraron repeticiones en los mantos; pero también por errores de procedimiento. En general, los valores de gas encontrados en Sogamoso están entre 0,2 y 6 pie $^{3}$ / ton, y son bajos si se comparan con carbones del mismo rango (bituminosos alto volátiles) y profundidades de alrededor de 50 m o más, medidos en Cesar-Ranchería en el caribe colombiano y en otras partes del mundo, donde se obtuvieron valores cercanos a $100 \mathrm{pie}^{3} /$ ton [13][14][8]

En Corrales, los contenidos de gas están entre 5 y 69 $\mathrm{pie}^{3} /$ ton, a profundidades entre 250 y 350 m con el gas desorbido representando más del 90\%. Estos valores también se consideran bajos para las profundidades alcanzadas. Para el municipio de Tasco, los valores encontrados están entre 50-100 $\mathrm{pie}^{3} /$ ton a profundidades que alcanzaron los $600 \mathrm{~m}$, lo que muestra un incremento significativo con respecto a los bloques ubicados en Sogamoso. En estas muestras no se midió gas residual y los valores de gas perdido están muy bajos, por lo que los valores reales podrían objetivamente estar cerca a los 150 pies $^{3} /$ ton. En Tasco-Paz de Río se encontraron valores totales entre 0-165 pies $^{3} /$ ton; los valores de gas residual se consideran bajos y en general los valores de todas las muestras deberían estar por encima de 100 pies $^{3} /$ ton porque el rango de los carbones es bituminoso medio volátil. En Socha se encontraron valores de contenido gas que están entre 0-275 $\mathrm{pie}^{3} /$ ton a profundidades entre 100 y 300 m. En Socotá se encontraron valores de gas que están entre 2-50 $\mathrm{pie}^{3} /$ ton a profundidades entre 400 y $600 \mathrm{~m}$. Los contenidos de gas superiores a $200 \mathrm{pie}^{3} /$ ton se consideran aceptables para las condiciones de la zona debido a la profundidad y al incremento del rango hacia el norte en que el rango de los mantos más inferiores es bituminoso medio volátil [8].

En general, los contenidos de gas en el área de SogamosoSocotá evidencian un aumento de sur a norte, lo que concuerda con el aumento del rango del carbón hacia el norte, donde se encuentran carbones de rango medio a bajo volátiles, que son coquizables. Generalmente, e aumento del rango del carbón se relaciona directamente con el incremento en el contenido de gas. En esta área, el rango del carbón y la reflectancia de la vitrinita se relacionan así: en la zona de Sogamoso, el rango del carbón es bituminoso alto volátil con valores de reflectancia de la vitrinita de 0,45-0,65\%, mientras que, en la parte norte, cerca a Socotá, el rango del carbón es bituminoso medio volátil, con valores de reflectancia de 
la vitrinita de 1-1,2\%, y con propiedades coquizables [6]. Estos valores de vitrinita están sobre los valores mínimos $(0,6-0,8 \%)$, a partir de los cuales se pueden generar grandes volúmenes de gas termogénico [15]. El aumento del rango de los carbones hacia el norte coincide no solamente con el aumento en el contenido de gas, sino también con la mayor subsidencia de la cuenca hacia el norte donde se encuentra el depocentro de la Cuenca del Cocuy [16][8].

\section{METODOLOGÍA}

De la base de datos de la Agencia Nacional de Minería se revisaron los contenidos de gas metano en porcentaje reportados durante las visitas de fiscalización desde al año 2017 hasta la fecha [17]. De acuerdo con la información disponible se decidió analizar la información de dos áreas carboníferas: 802 - Tunja - Paipa - Duitama, y 803 Sogamoso - Jericó. En total se analizaron y graficaron las mediciones adelantadas en 107 títulos mineros. Aunque en Boyacá se ha determinado 10 áreas carboníferas, solo se analizaron dos por la disponibilidad de datos y por la actividad minera [6]. Es evidente que la información existente puede mejorar y, que esta debe ser actualizada a medida que se generan las visitas.

Dicha información se organizó y tabulo en Excel. Posteriormente, se filtró la información para utilizar los datos de mayor utilidad. Los contenidos de gas con sus respectivas coordenadas se graficaron en el sistema de información geográfica (SIG). Se procesaron los datos mediante interpolación para resaltar las zonas más representativas en cuanto a riesgo de explosión. El software permite cargar cada punto correspondiente al título minero con su determinada información, y así implementar cada procesamiento y uso de las herramientas que este ofrece de cada punto de manera individual para obtener un resultado que, en conjunto, presente patrones sobre los cuales se pueda llegar a dar una interpretación concreta y fundamentada.

Durante el desarrollo de este estudio, se empleó el método Inverse Distance Weighted (IDW) con el fin de interpolar los valores más altos y más bajos, y así enfatizar en los puntos más cercanos; como resultado, los datos cercanos tendrán mayor influencia, y la superficie tendrá más detalles, a medida que aumenta la potencia, y los valores interpolados comienzan a ser cercanos al valor del punto de muestra. Lo que significa que, al especificar un valor más bajo de potencia, los puntos circundantes adquirirán más influencia que los que están más lejos, como resultado se obtiene una superficie más suave. Finalmente, se delimitaron las zonas más vulnerables por riesgo de explosión de gas metano.

A continuación, se genera una reclasificación sobre los rangos dados, donde se le asigna 1 y 2 para los rangos de 0 a $5 \%, 3$ y 4 para los rangos de 5 a $10.4 \%$, y 5 para los rangos mayores a $10 \%$; lo anterior, con el fin de dejar clases e intervalos definidos, y así lograr agrupar la mayor cantidad de datos en 5 tipos diferentes. En este caso, los valores que se encuentran dentro del grupo 1 , son de muy bajo riesgo; los del 2 son de bajo riesgo; los del 3, de mediano riesgo; del 4, de alto riesgo y; los de grupo 5 de muy alto riesgo.

Luego de tener los datos reclasificados, se empleó la herramienta de dissolve con el fin de simplificar los datos, teniendo en cuenta los que ya se tenían, esto para generar una nueva cobertura mediante la fusión de polígonos adyacentes con el mismo valor para un elemento especificado, y así obtener un solo polígono dependiendo el rango. A partir de lo anterior, se puede modificar el símbolo de los puntos de gas metano en cada título y asignarles un tamaño que incremente o disminuya proporcionalmente al porcentaje presentado, de tal manera que, se evidencie las zonas con mayor amenaza y se haga un close up sobre cada una e interpretarla de la mejor manera.

\section{RESULTADOS}

Las dos áreas relacionadas en el marco teórico se analizaron conjuntamente y se reportaron en un gran polígono que comprende las áreas 802 y 803 (Figura 2). 


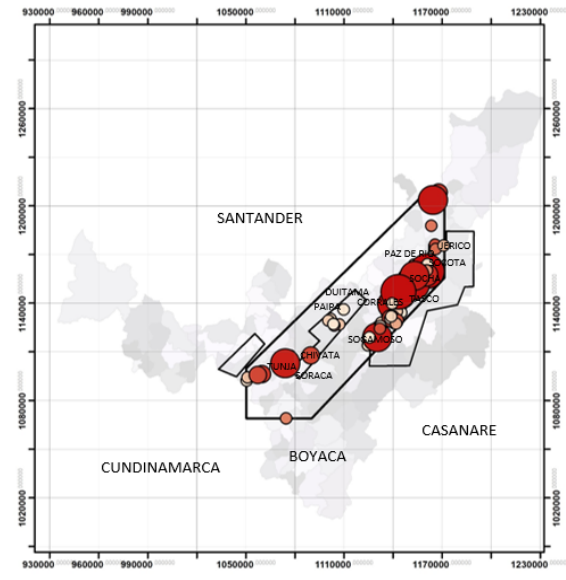

Figura 2. Ubicación del polígono del área de estudio.

En cada caso se introdujeron los datos de porcentaje de contenido de gas metano reportados durante las visitas. De la misma manera, se establecieron intervalos de porcentaje de 0 a 5 de 5 a 20 y mayores de 20 basados en las tres fases del gas metano; deflagrante, explosivo y asfixiante, respectivamente, donde las zonas más altas presentan tonalidades rojizas y anaranjadas para mostrar las zonas de mayor riesgo, tonalidad de amarillo para las zonas de mediano riesgo, y tonalidades verdosas para las zonas de bajo riesgo; gracias a estas convenciones, se puede determinar sobre qué zonas se implementarán medidas de mayor importancia a fin de prevenir accidentes, dependiendo del nivel de riesgo (Figura 3).

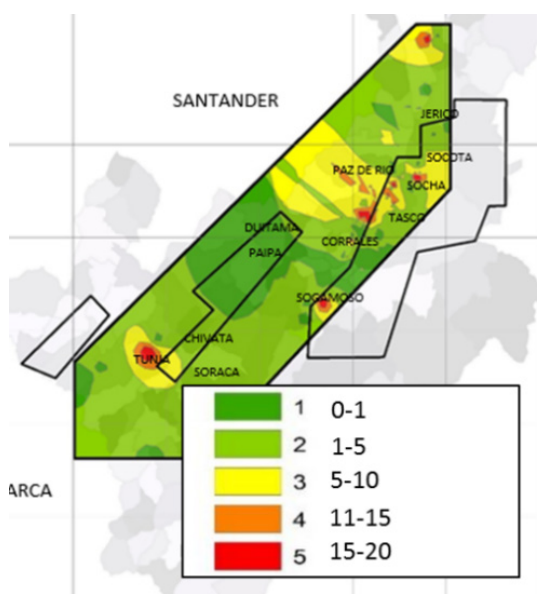

Figura 3. Mapa IDW de intervalos de contenido de gas del polígono de estudio.
Finalmente, los datos de contenido de gas se agruparon no por contenido de gas sino por el grado de riesgo dependiendo del contenido; donde grupo 1 son de muy bajo riesgo, los del grupo 2 son de bajo riesgo, los del grupo 3 de mediano riesgo, grupo 4 de alto riesgo y los de grupo 5 de muy alto riesgo. Como se fusionaron los de polígonos adyacentes con el mismo valor para poder evidenciar las zonas con mayor amenaza y facilitar la interpretación, se presentan las tres áreas carboníferas conjuntamente (Figura 4).

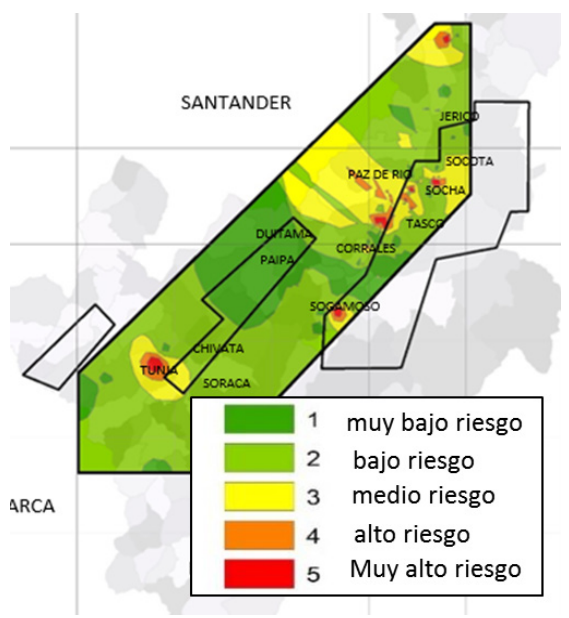

Figura 4. Mapa de reclasificación por riesgo de explosividad. Fuente: Los autores.

Después de tener los datos reclasificados se usó la herramienta de dissolve con el fin de simplificar los datos a partir de los que ya se tenían, esto para generar una nueva cobertura mediante la fusión de polígonos adyacentes con el mismo valor para un elemento especificado, y así obtener un solo polígono dependiendo el rango; con esta información se puede modificar símbolo de los puntos de gas metano en cada título y asignarles un tamaño que incremente o disminuya proporcionalmente al porcentaje presentado, esto para poder evidenciar las zonas con mayor amenaza y hacer un close up sobre cada una y llegar a interpretarla de la mejor manera. Como resultado se obtuvieron 3 zonas de amenaza (Figura 5) 


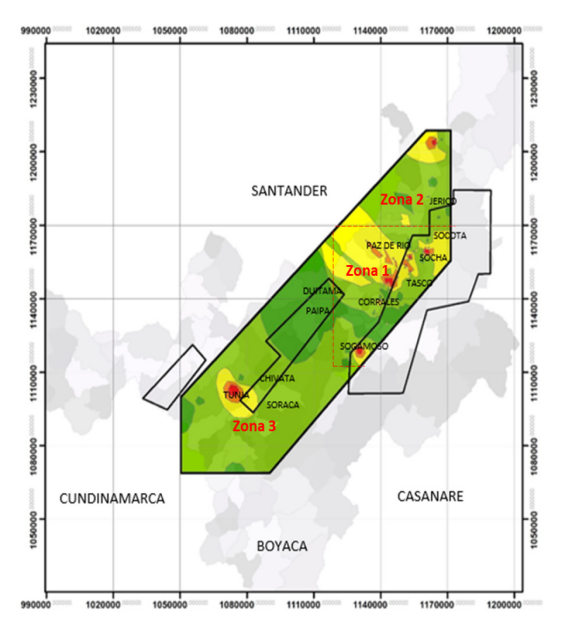

Figura 5. Mapa de Disolución de las tres zonas de amenaza.

\section{ANÁLISIS}

Con el mapa de dilución que permite resaltar mejor las zonas con mayor potencial o probabilidad que ocurra un accidente, se superpusieron dichas zonas con las áreas carboníferas mencionadas en el marco teórico (Figura 1). Finalmente, resultaron tres zonas las cuales se clasifican en orden dependiendo el riesgo, donde la zona 1 es la de mayor riesgo y la zona 3 la de menor riesgo.

Zona de Amenaza 1. Para la zona 1 o zona de mayor riesgo, los mayores porcentajes de metano están entren $20.9,20.8,20.4$, 18, 16, 12, 2.5, y $2.2 \%$; que corresponden con la parte central del área carbonífera 804- SogamosoJericó; específicamente, coincide con las explotaciones carboníferas de los municipios de tasco, Socha y Socotá (Figura 5). Los estudios de gas asociado al carbón en los que se mide el contenido de gas sobre corazones de perforación, y utilizando sistemas de desorción cánisters, han concluido que la zona de Socha-Socotá presenta altos contenidos de gas de hasta $250 \mathrm{pies}^{3} /$ ton a profundidades cercanas a $400 \mathrm{~m}$ de profundidad [5]. En esta zona son frecuentes los accidentes relacionados con la presencia de metano posiblemente originado por el incremento del rango del carbón en Boyacá hacia el norte, al punto que en esta zona los carbones son coquizables.
Zona de amenaza 2. Intermedio, los mayores porcentajes de metano reportados fluctúan entre 20.8 y 3.38 \%, que corresponden con la parte norte del área carbonífera 804Sogamoso-Jericó, en los alrededores del municipio de San Mateo en el norte de Boyacá (Figura 5). No se conocen resultados de los ensayos de desorción, pero como el rango del carbón mejora hacia el norte, se esperaría que el contenido del gas mejore proporcionalmente.

Zona de amenaza 3. Para la zona 3 o zona de riesgo menor, el mayor porcentaje de gas metano es del 19.4 \% y corresponde con el área 803 - Tunja-Paipa-Duitama (Figura 5). Los estudios de desorción realizados en la zona por el SGC, UPTC y la ANH muestran que los contenidos son bajos. Lo anterior podría estar relacionado con el fracturamiento de la zona y el alto buzamiento de los carbones, lo que facilitaría el escape del gas [9] [18].

A pesar de la frecuencia de los accidentes mineros relacionados con la presencia del gas metano, los estudios de desorción indican que los contenidos de gas en Boyacá no son altos porque no sobrepasan los $350 \mathrm{pies}^{3} /$ ton (10 $\mathrm{m}^{3}$ /ton) establecido por Naciones Unidas [19]; y que por lo tanto, no habría necesidad de drenar los macizos carboníferos antes de la explotación minera; por lo esta razón, los altos porcentajes de metano reportados y la frecuencia de los accidentes se relacionarían más con deficiencias en el diseño de la ventilación, condiciones mecánicas y circuitos eléctricos.

Se sugiere que, a ANNA, la plataforma digital que utiliza la Agencia Nacional de Minería (ANM) y que integra la información minero ambiental, se le pueda adicionar una sección que permita la actualización de la información en cada visita y, con el visor geográfico, tener la posibilidad de percibir estos patrones de riesgo, los cuales podrían servir de soporte al momento de crear las rutas de fiscalización.

\section{CONCLUSIONES}

El propósito principal de la investigación es zonificar el departamento de Boyacá por riesgo de explosión de gas metano en la minería del carbón, a partir de las 
mediciones en los frentes mineros con multidetectores durante las visitas de seguimiento y fiscalización.

Se confirmó la hipótesis de que el riesgo de accidente por presencia de gas metano es proporcional a los contenidos de gas reportados durante las visitas de fiscalización.

La zona de mayor riesgo de explosión por presencia de metano corresponde a la zona 3 o parte central de la zona carbonífera 801-Sogamoso-Jericó, entre los municipios de Tasco y Socotá, donde se ha reportado porcentajes entre 2 y $20,4 \%$ de metano.

El riesgo de la zona 3 se confirma por los relativamente altos contenidos de metano (pies 3 /ton) encontrados en los estudios de desorción, y por los frecuentes accidentes reportados en la zona.

Se concluye que los mayores porcentajes de metano encontrados entre Tasco y Socotá indican que el seguimiento por parte del estado y las nuevas rutas de fiscalización deberán dar prioridad a la parte central del área carbonífera 801.

Después de obtener los resultados se evidencia que el alto porcentaje de gas metano de algunos títulos puede presentar una influencia indirecta en los títulos circundantes, generando un efecto domino negativo; cabe aclarar que para estos estudios se necesitan de otro tipo de datos y un mayor número de muestras, ya sea para evidenciar la afectación a nivel del subsuelo, o para observar cómo se podría ver afectada la continuidad del yacimiento.

Como resultado de este proyecto se determinó que la información deberá ser ampliada y actualizada a medida que se generan las visitas, y así se podrá actuar con anticipación a los hechos. Igualmente, que la fiscalización debe dar prioridad a las zonas con mayor riesgo.

Así también, se concluye que la herramienta ANNA usada para le extracción minera, se puede actualizar para poder sistematizar la información en cada visita y, gracias al visor geográfico, tener la posibilidad de percibir estos patrones de riesgo, los cuales podrían servir de soporte en el momento de crear las rutas de fiscalización.

Por último, se afirma que sí estas medidas llegan a implementarse, se podría evitar y mitigar los accidentes producto de explosiones por gas metano en el departamento de Boyacá; por lo tanto, se reduciría el número de fallecidos y las pérdidas económicas.

\section{REFERENCIAS}

[1] Decreto 1886 (2015). Disponible en: https:// www.minminas.gov.co/documents/10180//235 17//36743-Decreto-1886-21Sep2015.

[2] J. Mariño, A. Castro, L. Mojica, A. Botero, D. Granados y C. Acuña (2015) "Desarrollo del CBM en Colombia," in Gas asociado al carbón (CBM o GMAC). Geología, contenidos, reservas, minería y posibilidades en Colombia, pp. 110-130, Tunja, Colombia:https://www.libreriadelau.com/ gas-asociado-al-carbon-cbm-o-gmac-geologiacontenidos-reservas-mineria-y-posibilidades-encolombia-universidad-pedagogica-y-tecnologicade-colombia-uptc-geologia/p

[3] UPME - Consorcio GEOIL- SERGEING- METANO CO. "Construcción de un modelo para Colombia de aprovechamiento u oxidación de gas metano de los ductos de ventilación (vam) de las minas subterráneas de carbón". 2017.

[4] C. Ortega-Ramos, F.-B.-M.-E. (2018). Boletín de Geología. "Evaluación del riesgo de explosividad del gas metano en minería subterránea de carbón, caso de la cuenca del Sinifaná, Colombia". Obtenido de: https://revistas.uis.edu.co/index.php/ revistaboletindegeologia/article/view/7961/829 2018.

[5] J. E. Mariño Martínez \& R. Chanci Bedoya, Gases de efecto invernadero: propuesta de cálculo del factor de emisiones de gas metano procedente de la extracción del carbón colombiano. Tunja: UPTC. 2019.

[6] G. Renzoni, 2006. Tareas para el desarrollo 
del carbón en Colombia. Obtenido de: http:// aplicaciones1.ingeominas.gov.co/sicat/html/ ConsultaBasica.aspx 2006.

[7] E. Arias, C. Beltrán, V. Gómez, N. Hernández, A. Rosas, y G. Tibaduiza, G. 1997. "Programa Normalización de Recursos y Reservas de carbón (Departamento de Boyacá)". ECOCARBÓN. 1997.

[8] J. Mariño \& L. Mojica, "Estados de la exploración y posibilidades de gas asociado al carbón (GAC) en Boyacá (COLOMBIA)". Revistas UIS. Obtenido de:https://revistas.uis.edu.co/index.php/ revistaboletindegeologia/article/view/3733/4160. 2012

[9] ANH - EAFIT. "Valoración del potencial exploratorio CBM en la cuenca carbonífera de Amagá y BoyacáCundinamarca (Formación Guaduas)". Convenio 014 de 2010. Bogotá. 2011.

[10] E. Carlier, y R. Camargo, Descripción litoestratigráfica y medición de volumen de gas metano asociado al carbón (GMAC) en núcleos de perforación en el pozo Chivatá-1 municipio de Chivatá, departamento de Boyacá. Trabajo de grado. UPTC Sogamoso, Ingeniería Geológica. Biblioteca UPTC, 2012.

[11] J. Fonseca y L. López, (2003) Determinación del bloque con mejores de posibilidades del gas asociado al carbón en la zona carbonífera ubicada al nororiente del municipio de Sogamoso. UPTC Sogamoso, Ingeniería Geológica.

[12] D. Pitre y H. Noppe, (2003) Determinación del bloque con mejores posibilidades del gas asociado al carbón en el sector SE de Sogamoso. Trabajo de grado. Sogamoso-Ingeniería Geológica. Biblioteca UPTC.

[13] Geomet, INC.1993. Proposal for Association Contract Coal Seam Methane Project CesarRancheria area Colombia. ECOPETROL. Bogotá. 1993.

[14] B.E. Law and D.D. Rice, 1993. "Hydrocarbons from Coal. Tulsa, Oklahoma, American Association of Petroleum Geologists, AAPG Studies in Geology" 38: 159-184. 1993.

[15] T. Moore, "General introduction to coal bed methane. In First annual Illinois Basin coalbed symposium", The Midwest PTTC Center. Evansville, Indiana, USA. 2004.

[16] A. Fabre, "La subsidencia de la Cuenca del Cocuy (Cordillera Oriental Colombiana) durante el Cretáceo y el Terciario Inferior". Geología Norandina No. 8, Bogotá 1983.

[17] ANM. Obtenido de: https://www.anm.gov.co/ sites/default/files/DocumentosAnm/estudio-casoexplosion-metano-y-polvo-carbon.pdf 2020.

[18] SGC. "Exploración de recursos energéticos, la exploración de gas metano asociado al carbón (GMAC o CBM) en un polígono de $200 \mathrm{~km} 2$, en los municipios de Guachetá, Ráquira". Obtenido de: httpshttps://catalogo.sgc.gov.co/cgi-bin/koha/ opac-detail.pl?biblionumber=49123. 2012.

[19] Naciones Unidas "Guía de mejores prácticas para un drenaje y uso eficaz del metano en las minas de carbón". Ginebra (Suiza) 2016. Obtenido de:

https://www.google.com/search?q=Naciones+Unid asGuia+de+mejores+practicas+para+un+drenaje+y +uso+eficaz+del+metano+en+las+minas+de+ carbon.+Ginebra\&oq=Naciones+UnidasGuia+de+ mejores+practicas+para+un+drenaje+y+uso+eficaz +del+metano+en+las+minas+de+carbon.+Ginebra \&aqs=chrome..69i57.1676j0j7\&sourceid=chrome $\& \mathrm{ie}=$ UTF-8 